\title{
ON APPROXIMATIVE SOLUTIONS OF OPTIMAL STOPPING PROBLEMS
}

\author{
ANDREAS FALLER * AND \\ LUDGER RÜSCHENDORF, ${ }^{* *}$ University of Freiburg
}

\begin{abstract}
In this paper we establish an extension of the method of approximating optimal discretetime stopping problems by related limiting stopping problems for Poisson-type processes. This extension allows us to apply this method to a larger class of examples, such as those arising, for example, from point process convergence results in extreme value theory. Furthermore, we develop new classes of solutions of the differential equations which characterize optimal threshold functions. As a particular application, we give a fairly complete discussion of the approximative optimal stopping behavior of independent and identically distributed sequences with discount and observation costs.
\end{abstract}

Keywords: Optimal stopping; max-stable distribution; Poisson process

2010 Mathematics Subject Classification: Primary 60G40; 62L15

\section{Introduction}

The theory of optimal stopping of independent and dependent sequences $X_{1}, \ldots, X_{n}$ is a classical subject of probability theory for which many open problems remain and new applications (for instance, in the area of financial mathematics) have been suggested. In a series of papers an approximation method was developed in order to solve approximatively optimal stopping problems for $X_{1}, \ldots, X_{n}$ by some limiting stopping problems for Poisson and related point processes (see Kühne and Rüschendorf (2000a), (2000b), (2003), (2004)). The basic assumption in this approach is convergence of the imbedded planar point process

$$
N_{n}=\sum_{i=1} \delta_{\left(i / n, X_{i}^{n}\right)} \stackrel{\mathrm{D}}{\rightarrow} N
$$

to some Poisson (or related) point process $N$. Here $X_{i}^{n}=\left(X_{i}-b_{n}\right) / a_{n}$ is a normalization of $X_{i}$ induced typically from the central limit theorem for maxima. For the limiting Poisson-type process $N$, which has accumulation points along a lower boundary curve, an optimal stopping problem in continuous time can be formulated.

The optimal solution for this limiting stopping problem is given by a threshold stopping time. The threshold function is determined by a first-order differential equation. This is in analogy to stopping problems for diffusion processes which typically lead to free boundary value problems with second-order differential equations for the stopping curve (the Stefan free boundary problem). The differential equation for the optimal threshold function in the Poisson case can be solved in several cases explicitly or numerically. Under some uniform

Received 16 November 2009; revision received 11 January 2011.

* Andreas Faller died unexpectedly on 30 June 2011.

** Postal address: Mathematical Stochastics, University of Freiburg, Eckerstr. 1, 79104 Freiburg, Germany.

Email address: ruschen@stochastik.uni-freiburg.de 
integrability and separation conditions, a differentiability condition for the intensity measure of $N$ as well as an asymptotic independence condition in the dependent case of the optimal stopping problem for $X_{1}, \ldots, X_{n}$ can be approximated by the optimal stopping problem for the limiting Poisson-type process.

In this paper we establish some relevant extensions of interest for this approximation method. In Section 2 we give a new and simplified derivation of the optimal stopping curve $u$ for the optimal stopping problem for continuous-time Poisson processes as above. These curves solve a differential equation of the form

$$
u^{\prime}(t)=-\int_{u(t)}^{\infty} G(t, y) \mathrm{d} y, \quad t \in[0,1), \quad u(1)=c,
$$

with some guarantee value $c \in \mathbb{R} \cup\{-\infty\}$. Here $G$ is defined explicitly via the intensity measure of $N$ and is called the 'intensity function'. For $c \in \mathbb{R},(1.1)$ has a unique solution and, thus, characterizes the optimal stopping curve. For $c=-\infty$, there may exist several solutions of (1.1). While the finite case $c \in \mathbb{R}^{1}$ has been dealt with in Kühne and Rüschendorf (2000a), the case where $c=-\infty$ has been left mostly open in the previous literature and has been dealt with only under an uniqueness assumption on (1.1). Based on our new derivation of the approximation result, we characterize the optimal stopping curve for $c=-\infty$ as the maximal solution of (1.1). We also establish some uniqueness criteria for (1.1) in the case $c=-\infty$. There are several interesting applications with $c=-\infty$ (see, e.g. the examples in Section 5 of this paper) which can be solved with our new extension of the approximation method.

The second main contribution of this paper concerns the differential equation (1.1), which characterizes optimal stopping boundaries. The classical results from differential equations for (1.1) concern the so-called separable case where $G(t, y)=a(t) b(y)$. However, even in this case the known characterization results for solutions are typically not explicit but need numerical tools. In this paper we introduce two interesting new classes of intensity functions $G$-not leading to the case of separate variables in (1.1)—which allow us to solve the differential equation (1.1) in 'explicit' form. For these classes of intensity functions, the so-called 'separation condition', which is needed in our approximation approach to optimal stopping problems, can be verified. In Section 4 we state an extension of the approximation theorem in Kühne and Rüschendorf (2004, Theorem 2.1) for the optimal stopping of dependent sequences. This is the second main ingredient of the approximation method. Our version gives a precise uniform integrability condition which allows us to also treat the case $c=-\infty$ (which was not included in previous results) and allows for more general filtrations.

As an application of our extensions, in Section 5 we discuss in fairly complete form the optimal stopping of independent and identically distributed (i.i.d.) sequences $\left(Z_{i}\right)$ with discount factors $\left(c_{i}\right)$ and observation costs $\left(d_{i}\right)$, i.e. of

$$
X_{i}=c_{i} Z_{i}+d_{i}
$$

Here $c_{i}$ and $d_{i}$ fulfill some criteria to ensure point process convergence, and $\left(Z_{i}\right)$ is an i.i.d. sequence in the maximum domain of an extreme value distribution $\Gamma, \Phi_{\alpha}$, or $\Psi_{\alpha}$. The new results on the solution of the optimality equation in (1.1) and the inclusion of the case $c=-\infty$ allow us to complete some partial results on this problem in Kühne and Rüschendorf (2000b). This kind of stopping problem was first considered in the i.i.d. case without discount and observation cost in Kennedy and Kertz (1990), (1991).

It has been observed in several papers in the literature that optimal stopping problems may have an easier solution in a related form for Poisson numbers of points, as, for instance, in the 
classical house selling problem (see Chow et al. (1971), Bruss and Rogers (1991), and Gnedin and Sakaguchi (1992)). The approach extended in this paper makes this method applicable to a wider class of examples. Based on the new results in this paper, an interesting extension to multistopping problems has recently been proposed in Faller and Rüschendorf (2011). Several details and proofs in this paper have been omitted to save space; they can be found in the dissertation of Faller (2009), on which this paper is based.

\section{Optimal stopping of Poisson processes}

We consider optimal stopping of a Poisson process $N=\sum_{k} \delta_{\left(\tau_{k}, Y_{k}\right)}$ in the plane restricted to some set

$$
M_{f}=\{(t, x) \in[0,1] \times \overline{\mathbb{R}}: x>f(t)\},
$$

where $f:[0,1] \rightarrow \mathbb{R} \cup\{-\infty\}$ is a continuous function describing the lower boundary of $N$. We consider Poisson processes restricted to $M_{f}$ which may have infinite intensity along the lower boundary $f$. We assume that the intensity measure $\mu$ of $N$ is a Radon measure on $M_{f}$ with the topology on $M_{f}$ induced by the usual topology on $[0,1] \times \overline{\mathbb{R}}$. Thus, any compact set $A \subset M_{f}$ has only finitely many points. By convergence in distribution, i.e. $N_{n} \stackrel{\mathrm{D}}{\rightarrow} N$ on $M_{f}$, we mean convergence in distribution of the restricted point processes.

We generally assume the boundedness condition:

(B) $\mathrm{E}\left(\sup _{k} Y_{k}\right)^{+}<\infty$.

Let $\mathcal{A}_{t}=\sigma\left(N\left(\cdot \cap[0, t] \times \overline{\mathbb{R}} \cap M_{f}\right)\right), t \in[0,1]$, denote the relevant filtration of the point process $N$. A stopping time $T: \Omega \rightarrow[0,1]$ for $N$ is a stopping time with respect to the filtration $\left(\mathcal{A}_{t}\right)$, i.e. $\{T \leq t\} \in \mathcal{A}_{t}, t \in[0,1]$. In general, $N$ may have multiple points. Denote by $\bar{Y}_{T}:=\sup \left\{Y_{k}: T=\tau_{k}\right\}, \sup \varnothing:=-\infty$, the reward with respect to the stopping time $T$. For any guarantee value $x \in[c, \infty], c:=f(1)$, define the optimal stopping curve with respect to $x$ by

$$
\begin{aligned}
u(t, x) & :=\sup \left\{\mathrm{E}\left[\bar{Y}_{T} \vee x\right]: T>t \text { a stopping time }\right\}, \quad t \in[0,1), \\
u(1, x) & :=\mathrm{E}\left[\bar{Y}_{1} \vee x\right]
\end{aligned}
$$

In contrast to Kühne and Rüschendorf (2000a) we consider stopping times $T>t$ and introduce a guarantee value. This has some technical advantages with respect to the continuity properties and leads to some changes in the optimal stopping time formulae. For notational convenience, we write

$$
u(t):=u(t, c), \quad t \in[0,1] .
$$

Every instance of $u$ without arguments is to be understood as $u(\cdot, c)$. The critical point of $N$ for $x$ is given by

$$
t_{0}(x):=\inf \left\{t \in[0,1]: N\left((t, 1] \times(x, \infty] \cap M_{f}\right)=0, \text { P-almost surely }\right\}, \quad \text { inf } \varnothing:=1 .
$$

The following lemma gives some basic properties of the optimal stopping curve $u$.

Lemma 2.1. (a) For any $x \in[c, \infty]$, the optimal stopping curve $u(\cdot, x)$ is right continuous on the interval $\{t \in[0,1]: u(t, x)>-\infty\}$.

(b) For $x, y \in \mathbb{R}, x \leq y$,

$$
0 \leq u(t, y)-u(t, x) \leq y-x, \quad t \in[0,1] .
$$


(c) For $x \in[c, \infty], x>-\infty$,

$$
t_{0}(x)=\inf \{t \in[0,1]: u(t, x)=x\}, \quad \inf \varnothing:=1 .
$$

Proof. For the proof, see Faller (2009).

In order to identify the optimal stopping curve $u$ for the stopping of the Poisson process $N$, in the following we generally assume that $t_{0}(c)=1$, and that the intensity measure $\mu$ of $N$ is a Radon measure on $M_{f}$ and Lebesgue continuous with density $g$.

An important condition is the separation condition:

(S) assume that $u>f$ on $[0,1)$.

If $c=-\infty$ then (S) implies the right continuity of $u$ on $[0,1]$ and the validity of Lemma 2.1(c) for $x=-\infty$ also. By our general assumption $t_{0}(c)=1$, the separation condition (S) is in general satisfied for $c \in \mathbb{R}$ if $f \leq c$.

With the additional guarantee parameter $x$, Theorem 2.5(a) of Kühne and Rüschendorf (2000a) in our slightly modified form can be formulated as follows.

Theorem 2.1. (Existence and uniqueness of an optimal stopping time.) Let $N$ satisfy conditions $(B)$ and $(S)$. Then, for any $x \geq c$, the optimal stopping curve $u(\cdot, x):[0,1] \rightarrow[x, \infty)$ is continuous on $[0,1]$. Furthermore,

$$
u(t, x)=\mathrm{E}\left[\bar{Y}_{T(t, x)} \vee x\right], \quad t \in[0,1],
$$

where the optimal stopping time $T(t, x)$ at time $t$ is given by

$$
T(t, x)=\inf \left\{\tau_{k}>t: Y_{k}>u\left(\tau_{k}, x\right)\right\}, \quad \inf \varnothing:=1 .
$$

Thus, $T(0, x)$ is an optimal stopping time for $N, x$ at time 0. It is $\mathrm{P}$-almost surely (P-a.s.) unique.

Note that, by condition (S), in the $c=-\infty$ case we have $\mathrm{P}(T(t, c)<1)=1$, or, equivalently, $\mu\left((t, 1] \times \mathbb{R} \cap M_{u}\right)=\infty$ for all $t \in[0,1)$.

In the following we want to characterize the optimal stopping curve $u$ by a differential equation. The following lemma will be needed in the $c=-\infty$ case.

Lemma 2.2. Let $N$ satisfy the boundedness condition (B), and define

$$
v(t):=\lim _{x \downarrow c} u(t, x), \quad t \in[0,1] .
$$

If, for some continuous function $w:[0,1) \rightarrow \mathbb{R}, v \geq w>f$ holds on $[0,1)$, then $v=u$. In particular, the separation condition $(S)$ is satisfied.

Proof. For $t \in[0,1]$, let $T_{v}(t):=\inf \left\{\tau_{k}>t: Y_{k}>v\left(\tau_{k}\right)\right\}$, inf $\varnothing:=1$, denote the threshold stopping time of $v$. This is a stopping time for $N$ since $v \geq w>f$ on $[0,1)$. Then $u \leq v \leq u(\cdot, x)$ and, thus, $T_{v}(t) \leq T(t, x)$. Furthermore, $\bar{Y}_{T(t, x)} \vee \bar{x} \rightarrow \bar{Y}_{T_{v}(t)} \vee c$, P-a.s. for $x \downarrow c$. This follows from our modified definition of $T(t, x)=\inf \left\{\tau_{k}>t: Y_{k}>u\left(\tau_{k}, x\right)\right\}$ (in comparison to the usual ' $\geq$ ' definition) and the fact that the thresholds $u(\cdot, x)$ converge to $v$. Thus, by Fatou's lemma, it follows that

$$
u(t) \leq v(t)=\lim _{x \downarrow c} u(t, x)=\lim _{x \downarrow c} \mathrm{E}\left[\bar{Y}_{T(t, x)} \vee x\right] \leq \mathrm{E}\left[\bar{Y}_{T_{v}(t)} \vee c\right] \leq u(t) .
$$


For any continuous function $v:[0,1] \rightarrow \mathbb{R} \cup\{-\infty\}$ with $v>f$ on $[0,1)$ and threshold stopping times

$$
T_{v}(t):=\inf \left\{\tau_{k}>t: Y_{k}>v\left(\tau_{k}\right)\right\}, \quad \inf \varnothing:=1,
$$

for $t \in[0,1)$, the Poisson assumption allows us to calculate the joint distribution of $\left(T_{v}(t), \bar{Y}_{T_{v}(t)}\right)$. By standard arguments for Poisson processes we obtain the following result.

Lemma 2.3. Let $N$ satisfy condition $(B)$. Then the distribution of $\left(T_{v}(t), \bar{Y}_{T_{v}(t)}\right)$ on $[0,1) \times \mathbb{R}$ has Lebesgue density

$$
F_{t}(s, z):= \begin{cases}\mathrm{e}^{-\mu\left((t, s] \times \mathbb{R} \cap M_{v}\right)} g(s, z) \chi_{M_{v}}(s, z) & \text { if } s \in(t, 1), \\ 0 & \text { if } s \in[0, t]\end{cases}
$$

In the sequel we will need the following differentiability condition on the density $g$ of $\mu$.

(D) Assume that there exists a version of the density $g$ of $\mu$ on $M_{f}$ such that the intensity function

$$
G(t, y):=\int_{y}^{\infty} g(t, z) \mathrm{d} z
$$

is continuous on $M_{f} \cap[0,1) \times \mathbb{R}$. Furthermore, assume that $\lim _{y \rightarrow \infty} y G(t, y)=0$ for all $t \in[0,1)$.

Based on Lemma 2.3, we now prove that, for $x \in[c, \infty)$, the optimality equation for a threshold function $v$, i.e.

$$
v(t)=\mathrm{E}\left[\bar{Y}_{T_{v}(t)} \vee x\right], \quad t \in[0,1), \quad v(1)=x,
$$

which, by Theorem 2.1, is fulfilled in particular for the optimal stopping curve $u(\cdot, x)$, is essentially equivalent to a first-order differential equation:

$$
v^{\prime}(t)=-\int_{v(t)}^{\infty} G(t, y) \mathrm{d} y, \quad t \in[0,1), \quad v(1)=x .
$$

In order to apply the differentiation and integration rules needed in the proof of this equivalence, we assume that the differentiability condition (D) holds. In the following we give a simplified proof of Theorem 2.5 of Kühne and Rüschendorf (2000a) and add essential information to the important $c=-\infty$ case. In this case a solution of the differential equation (2.3) does not need to satisfy the optimality equation (2.2), but we give a formula for the difference between $v(t)$ and the expected value $\mathrm{E} \bar{Y}_{T_{v}(t)}$, which will be used later to derive uniqueness results for the differential equation (2.3).

Proposition 2.1. (Equivalence of the optimality equation and differential equation.) Let $N$ satisfy conditions $(B)$ and $(D)$, and let $v:[0,1] \rightarrow \mathbb{R} \cup\{-\infty\}$ be continuous with $v>f$ on $[0,1)$ and $x \in[c, \infty)$.

(a) If $v$ satisfies the optimality equation (2.2) then it also satisfies the differential equation (2.3).

(b) If $x \in \mathbb{R}$ and $v$ satisfies the differential equation (2.3), then $v$ satisfies the optimality equation (2.2). 
(c) If $x=-\infty$ and $v$ satisfies the differential equation (2.3), then, for $t \in[0,1)$,

$$
v(t)-\lim _{s \uparrow 1} v(s) \mathrm{e}^{-\mu\left((t, s] \times \mathbb{R} \cap M_{v}\right)}=\mathrm{E} \bar{Y}_{T_{v}(t)} .
$$

As $v$ is assumed to be continuous and $v(1)=-\infty$, this implies that $v(t) \leq \mathrm{E} \bar{Y}_{T_{v}(t)}$.

Proof. We will make use of the partial integration formula which states that, for $p \geq 0$ measurable satisfying $\int_{a}^{\infty} z p(z) \mathrm{d} z<\infty$ and $\lim _{y \rightarrow \infty} y \int_{y}^{\infty} p(z) \mathrm{d} z=0$ for $a \in \mathbb{R}$,

$$
\int_{a}^{\infty} z p(z) \mathrm{d} z=a \int_{a}^{\infty} p(z) \mathrm{d} z+\int_{a}^{\infty} \int_{y}^{\infty} p(z) \mathrm{d} z \mathrm{~d} y .
$$

(a) For $t<1$, with $T(t):=T_{v}(t)$,

$$
v(t)=\mathrm{E}\left[\bar{Y}_{T(t)} \vee x\right]=\mathrm{E}\left[\bar{Y}_{T(t)} \chi_{\{T(t)<1\}}\right]+x \mathrm{P}(T(t)=1) .
$$

Since $v(t)>-\infty$ for $t<1$, we obtain $\mathrm{P}(T(t)=1)=0$ if $x=-\infty$. Without loss of generality, in the $x>-\infty$ case we can assume that $x=0$ (by shifting the point process and $v$ by $-x)$. Consequently, we obtain, by (2.5),

$$
\begin{aligned}
v(t) & =\mathrm{E}\left[\bar{Y}_{T(t)} \chi_{\{T(t)<1\}}\right] \\
& =\int_{t}^{1} \int_{v(s)}^{\infty} z g(s, z) \mathrm{d} z \mathrm{e}^{-\mu\left((t, s] \times \mathbb{R} \cap M_{v}\right)} \mathrm{d} s \\
& =\int_{t}^{1}\left(v(s) h(s)+\int_{v(s)}^{\infty} \int_{y}^{\infty} g(s, z) \mathrm{d} z \mathrm{~d} y\right) \mathrm{e}^{-\mu\left((t, s] \times \mathbb{R} \cap M_{v}\right)} \mathrm{d} s,
\end{aligned}
$$

where $h(s):=\int_{v(s)}^{\infty} g(s, z) \mathrm{d} z=G(s, v(s))$.

By condition (D) and the continuity of $v, h$ is continuous. For $f(s, t)$ differentiable in $s$ and continuous in $t$,

$$
\frac{\mathrm{d}}{\mathrm{d} t} \int_{t}^{1} f(s, t) \mathrm{d} s=\int_{t}^{1} \frac{\mathrm{d}}{\mathrm{d} t} f(s, t) \mathrm{d} s-f(t, t) .
$$

Since $\mu\left((t, s] \times \mathbb{R} \cap M_{v}\right)=-\int_{t}^{s} \int_{v(r)}^{\infty} g(r, z) \mathrm{d} z \mathrm{~d} r=-\int_{t}^{s} h(r) \mathrm{d} r$ is differentiable in $t$ with derivative $h(t)$, we obtain

$$
v^{\prime}(t)=v(t) h(t)-\left(v(t) h(t)+\int_{v(t)}^{\infty} \int_{y}^{\infty} g(t, z) \mathrm{d} z \mathrm{~d} y\right)=-\int_{v(t)}^{\infty} G(t, y) \mathrm{d} y .
$$

(b) Let $t<r \leq 1$. Similarly as in (a) and using condition (D), we obtain

$$
\begin{aligned}
\mathrm{E}\left[\bar{Y}_{T(t)} \chi_{\{T(t)<r\}}\right]= & \int_{t}^{r}\left(v(s) h(s)+\int_{v(s)}^{\infty} \int_{y}^{\infty} g(s, z) \mathrm{d} z \mathrm{~d} y\right) \mathrm{e}^{-\mu\left((t, s] \times \mathbb{R} \cap M_{v}\right)} \mathrm{d} s \\
= & \int_{t}^{r}\left(v(s) h(s)-v^{\prime}(s)\right) \mathrm{e}^{-\mu\left((t, s] \times \mathbb{R} \cap M_{v}\right)} \mathrm{d} s \\
= & \int_{t}^{r} v(s) h(s) \mathrm{e}^{-\mu\left((t, s] \times \mathbb{R} \cap M_{v}\right)} \mathrm{d} s \\
& -\left(\left[v(s) \mathrm{e}^{-\mu\left((t, s] \times \mathbb{R} \cap M_{v}\right)}\right]_{t}^{r}+\int_{t}^{r} v(s) h(s) \mathrm{e}^{-\mu\left((t, s] \times \mathbb{R} \cap M_{v}\right)} \mathrm{d} s\right) \\
= & v(t)-v(r) \mathrm{e}^{-\mu\left((t, r] \times \mathbb{R} \cap M_{v}\right)} .
\end{aligned}
$$


With $r=1$ and using the fact that $\mathrm{P}(T(t)=1)=\mathrm{P}\left(N\left((t, 1] \times \mathbb{R} \cap M_{v}\right)=0\right)=$ $\mathrm{e}^{-\mu\left((t, 1] \times \mathbb{R} \cap M_{v}\right)}$, we obtain (b) as $v(1)=x$.

(c) For $t<1$, as in (b), using Fatou's lemma, we obtain

$$
-\infty<v(t) \leq \underset{r \uparrow 1}{\lim \sup } \mathrm{E}\left[\bar{Y}_{T(t)} \chi_{\{T(t)<r\}}\right] \leq \mathrm{E}\left[\bar{Y}_{T(t)} \chi_{\{T(t)<1\}}\right]
$$

Thus, $\bar{Y}_{T(t)} \chi_{\{T(t)<1\}}$ is integrable. This implies uniform integrability of $\left\{\bar{Y}_{T(t)} \chi_{\{T(t)<r\}} \mid 0<\right.$ $r<1\}$, and, thus, convergence of the expectations. Furthermore, from the proof of (b), it follows by condition (B) that $\mu\left((t, 1] \times \bar{R} \cap M_{v}\right)=\infty$ as $v(r) \downarrow-\infty$ for $r \uparrow 1$. As $\mathrm{P}(T(t)=1)=\mathrm{P}\left(N\left((t, 1] \times \bar{R} \cap M_{v}\right)=0\right)=\mathrm{e}^{-\mu\left((t, 1] \times \overline{\mathbb{R}} \cap M_{v}\right)}=0$, we obtain $T(t)<1$, P-a.s. and, thus,

$$
v(t) \leq v(t)-\lim _{s \uparrow 1} v(s) \mathrm{e}^{-\mu\left((t, s] \times \mathbb{R} \cap M_{v}\right)}=\mathrm{E} \bar{Y}_{T_{v}(t)} .
$$

Based on the equivalence in Proposition 2.1, we now establish that the optimal stopping curve can be described as the solution of the first-order differential equation (2.3). We will see that the separation condition ( $\mathrm{S}$ ) is equivalent to the existence of a solution $v>f$ on $[0,1)$ with $v(1)=c$. This also holds in the $c=-\infty$ case. We first treat the case of a finite guarantee value.

Proposition 2.2. Let $x \in[c, \infty) \cap \mathbb{R}$, and let $N$ satisfy conditions $(B)$ and $(D)$.

(a) If $v_{1}, v_{2}:[0,1] \rightarrow \mathbb{R}$ with $v_{i}>f$ on $[0,1)$ are solutions of the differential equation (2.3), then $v_{1}=v_{2}$.

(b) If a solution $v:[0,1] \rightarrow \mathbb{R}$ of $(2.3)$ exists such that $v>f$ on $[0,1)$ then $u(\cdot, x)=v$. In particular, if $x=c$ then the separation condition $(S)$ is satisfied.

Proof. (a) See Kühne and Rüschendorf (2000a, p. 310).

(b) By Proposition 2.1(b), $v$ satisfies the optimality equation (2.2). By the definition of $u(\cdot, x), u(t, x) \geq \mathrm{E}\left[\bar{Y}_{T_{v}(t)} \vee x\right]=v(t)>f(t)$ for all $t \in[0,1)$. By Theorem 2.1 and Proposition 2.1(a), $u(\cdot, x)$ solves (2.3) and by part (a) we thus obtain $u(\cdot, x)=v$.

In contrast to the case of a finite guarantee value $x$, uniqueness of the solutions of (2.3) does not hold for $x=-\infty$. The following theorem identifies the optimal stopping curve in the set of all solutions of (2.3) as the largest one. It also gives a criterion for uniqueness.

Theorem 2.2. Let $c=x=-\infty$, and let $N$ satisfy conditions $(B)$ and $(D)$. Also, let $v:[0,1] \rightarrow$ $\mathbb{R} \cup\{-\infty\}$ be a solution of the differential equation (2.3) with $v>f$ on $[0,1)$. Then the following statements hold.

(a) We have $v \leq u$. In particular, the separation condition $(S)$ is satisfied and so $u$ is also a solution of (2.3).

(b) If, for some function $b:[0,1) \rightarrow \mathbb{R}$, we have $u \leq b$ (as, e.g. for $b(t):=\mathrm{E}\left[\sup _{\tau_{k}>t} Y_{k}\right]$ ) and

$$
\liminf _{t \uparrow 1} \frac{v(t)}{b(t)}<\infty,
$$

then $u=v$. If (2.6) holds with $v$ replaced by $f$ then the solution of (2.3) is uniquely determined. 
(c) Let $u_{s}$ denote the optimal stopping curve of $N_{s}:=N\left(\cdot \cap[0, s] \times \overline{\mathbb{R}} \cap M_{f}\right)$, let $b:[0,1) \rightarrow$ $\mathbb{R}$ satisfy $u \leq b$, and assume that, for every $s \in(1-\varepsilon, 1)$ with some $\varepsilon>0$, there exists a function $a_{s}:[0, s) \rightarrow \mathbb{R}$ with $f<a_{s} \leq u_{s}$ on $[0, s)$ such that

$$
\liminf _{t \uparrow 1} \frac{\lim \sup _{s \uparrow 1} a_{s}(t)}{b(t)}<\infty .
$$

Then the solution of (2.3) is unique.

Proof. (a) The proof follows from Proposition 2.1(c).

(b) By Lemma 2.1, $u$ is continuous and, therefore, $u$ solves (2.3). If $v \neq u$ then, as in the proof of Proposition 2.2, it follows that $u>v$ and $u^{\prime} \geq v^{\prime}$ on $[0,1)$. With $w(s):=u(s)-v(s)$ for $s \in[0,1)$ we have

$$
w^{\prime}(s)=u^{\prime}(s)-v^{\prime}(s)=\int_{v(s)}^{u(s)} \int_{y}^{\infty} g(s, x) \mathrm{d} x \mathrm{~d} y \geq w(s) \int_{u(s)}^{\infty} g(s, x) \mathrm{d} x,
$$

and, thus,

$$
\frac{\partial}{\partial s} \log (w(s))=\frac{w^{\prime}(s)}{w(s)} \geq \int_{u(s)}^{\infty} g(s, x) \mathrm{d} x .
$$

By integration we obtain $w(t) \geq w(0) \mathrm{e}^{\mu\left([0, t] \times \mathbb{R} \cap M_{u}\right)}$. Since, for $v=u$, equality holds in (2.4), we obtain

$$
\lim _{t \uparrow 1} u(t) \mathrm{e}^{-\mu\left([0, t] \times \mathbb{R} \cap M_{u}\right)}=0,
$$

and, thus,

$$
\frac{v(t)}{u(t)}-1=-\frac{w(t)}{u(t)} \geq w(0) \frac{1}{-u(t) \mathrm{e}^{-\mu\left([0, t] \times \mathbb{R} \cap M_{u}\right)}} \rightarrow \infty \quad \text { for } t \uparrow 1 .
$$

Since $u(t) \leq b(t)$, it follows that

$$
\frac{v(t)}{b(t)} \geq \frac{v(t)}{u(t)} \rightarrow \infty \quad \text { as } t \uparrow 1,
$$

contradicting our assumption. Thus, $v$ is the optimal stopping curve. If (2.6) holds for $f$ then it also holds for any solution $v>f$. Thus, uniqueness follows.

(c) See Faller (2009).

To verify the separation condition (S) and, thus, the existence of a solution of (2.3) (which is an assumption of Theorem 2.2), or to construct the functions $a_{s}$ used in part (c) of Theorem 2.2, we can use a comparison argument given in the following proposition.

Proposition 2.3. Let $N$ and $N_{*}$ be Poisson processes on $M_{f}$ which satisfy conditions $(B)$ and $(D)$, with intensity functions $G$ and $G_{*}$ and optimal stopping curves $u(t, x)$ and $u_{*}(t, x)$. Furthermore, let $N_{*}$ satisfy condition $(S)$, and let $u(\cdot, x)>f$ for all $x>c$. Then, for any $s \in$ $[0,1), G \geq G_{*}$ on $[s, 1) \times \mathbb{R} \cap M_{f}$ implies that $u(t, x) \geq u_{*}(t, x)$ for all $(t, x) \in[s, 1] \times[c, \infty]$. In particular, if $G \geq G_{*}$ then condition $(S)$ is also satisfied for $N$.

Proof. Assume first that $x \in \mathbb{R}$. For any $t \in[s, 1)$ with $u(t, x)<u_{*}(t, x)$, it holds that $u^{\prime}(t, x) \leq u_{*}^{\prime}(t, x)$ since

$$
u^{\prime}(t, x)=-\int_{u(t, x)}^{\infty} G(t, y) \mathrm{d} y \leq-\int_{u_{*}(t, x)}^{\infty} G(t, y) \mathrm{d} y \leq-\int_{u_{*}(t, x)}^{\infty} G_{*}(t, y) \mathrm{d} y=u_{*}^{\prime}(t, x) .
$$

Assume that, for some $r \in[s, 1), u(r, x)<u_{*}(r, x)$. 
Since $u(1, x)=u_{*}(1, x)=x$, there exists a $t_{0} \in(r, 1]$ such that $u\left(t_{0}, x\right)=u_{*}\left(t_{0}, x\right)$ and $u(t, x)<u_{*}(t, x)$ for all $t \in\left[r, t_{0}\right)$. This implies that

$$
u\left(t_{0}, x\right)-u(r, x)=\int_{r}^{t_{0}} u^{\prime}(t, x) \mathrm{d} t \leq \int_{r}^{t_{0}} u_{*}^{\prime}(t, x) \mathrm{d} t=u_{*}\left(t_{0}, x\right)-u_{*}(r, x),
$$

and, thus, $u(r, x) \geq u_{*}(r, x)$.

In the $x=-\infty$ case we obtain, from Lemma 2.2, $u \geq u_{*}>f$ on $[s, 1)$.

For some applications of this comparison principle, see Faller (2009).

Example 2.1. Let the intensity function be of the form $G(t, y)=A(t) \mathrm{e}^{-B(t) y}$ on $[0,1) \times$ $(-\infty, \infty]$ with continuous functions $A, B:[0,1) \rightarrow \mathbb{R}$ such that $A \geq 0, A(t)>0$ for large $t$, $\int_{0}^{1} A(t) \mathrm{d} t<\infty$, and $B>0$ is bounded such that $\liminf _{t \uparrow 1} B(t)>0$. Then we can compare $G, G_{*}$, i.e.

$$
G(t, y) \geq G_{*}(t, y):= \begin{cases}A(t) \mathrm{e}^{-M y} & \text { if } y \geq 0, \\ A(t) \mathrm{e}^{-m y} & \text { if } y<0,\end{cases}
$$

where $M:=\sup B$ and $m:=\inf B$. Thus, by Proposition 2.3 and using the terminology of Theorem 2.2(c), we obtain

$$
u_{s}(t) \geq a_{s}(t):=u_{*, s}(t)=\frac{1}{m} \log \left(\frac{1}{d}\left(1-\exp \left(-d \int_{t}^{s} A(r) \mathrm{d} r\right)\right)\right)
$$

with $d:=1-m / M>0$ for large enough $t<s$. Similarly, by estimating $G$ from above,

$$
u(t) \leq b(t):=\frac{1}{M} \log \left(\frac{1}{d^{\prime}}\left(1-\exp \left(-d^{\prime} \int_{t}^{1} A(r) \mathrm{d} r\right)\right)\right)
$$

with $d^{\prime}:=1-m / M<0$. This implies that condition (2.7) holds as $\lim _{x \downarrow 0} \log (1-$ $\left.\mathrm{e}^{-x}\right) / \log \left(\mathrm{e}^{v x}-1\right)<\infty$ for any $v>0$. Consequently, uniqueness of the solutions of (2.3) follows.

With $w(t):=\mathrm{e}^{u(t)}$ we find as a particular consequence that the differential equation

$$
w^{\prime}(t)=-A(t) w(t)^{1-B(t)}, \quad t \in[0,1), \quad w(1)=0,
$$

has a unique solution $w$ such that $w>0$ on $[0,1)$.

\section{Explicit solutions of the optimality equation}

In Section 2 the optimal threshold function was characterized by the differential equation (2.3), which, by the results in Section 2, we also call the optimality equation. In the case where the intensity function $G$ is separable, i.e. $G(t, y)=a(t) H(y)$, a characterization of the existence of solutions is given by a classical result on differential equations in separate variables (see Kühne and Rüschendorf (2000a, Proposition 2.6)). However, note that even in this case the characterization is in general far from an 'explicit' form of the solution. The second main point in this paper is the introduction of some new classes of intensity functions $G(t, y)$ which allow us to establish 'explicit' solutions of the optimality equation (2.1). An important class of applications of this development is given in Section 5 to optimal stopping of i.i.d. sequences 
with discount and observation costs. For further interesting applications of this development to a general treatment of multistopping problems, see Faller and Rüschendorf (2011).

In the following we will introduce two classes of intensity functions, which allow us to give an explicit form for the solutions of (2.3). These intensity functions are of the form

$$
G(t, y)=H\left(\frac{y}{v(t)}\right) \frac{v^{\prime}(t)}{v(t)}
$$

and

$$
G(t, y)=H(y-v(t)) v^{\prime}(t) .
$$

It should be noted that the functions $v$ used here are different from the threshold functions used in the last paragraph. The function $v$ in the representation of $G(t, y)$ is in general not unique. The main reason for introducing the type of representations given in (3.1) and (3.2) is that they imply that $v$ is up to a normalization concerning the boundary value a solution of the optimality equation (2.3). Thus, (3.1) and (3.2) are particularly useful representations of $G$ concerning solutions of (2.3). To see this connection, note that the optimality equation for $u$ can be written by substitution in the equivalent forms:

$$
u^{\prime}(t)=-\int_{u(t)}^{\infty} G(t, y) \mathrm{d} y=-\int_{1}^{\infty} G(t, y u(t)) u(t) \mathrm{d} y=-\int_{0}^{\infty} G(t, y+u(t)) \mathrm{d} y .
$$

In both representations (3.1) and (3.2) $v$ is therefore verified to be up to a normalizing constant and up to the boundary condition a solution of (2.3). This crucial observation motivates the representations in (3.1) and (3.2). We will see in the following that, for both (3.1) and (3.2), under some conditions on $H, v$ explicit solutions of the essential differential equation (2.3) can be found for any boundary value. This needs a detailed study of several cases.

Let $f:[0,1] \rightarrow \mathbb{R} \cup\{-\infty\}$ be a continuous lower boundary function, and, as before, let $c:=f(1)$. Let $N$ be a Poisson process on $M_{f}$ with intensity function $G$ which satisfies conditions (B) and (D).

\subsection{First class of intensity functions}

Let $f=a v$ on $[0,1)$ with $a \in \mathbb{R} \cup\{-\infty\}$ and a monotone $C^{1}$-function $v:[0,1) \rightarrow \mathbb{R}$, $v>0$, and assume that $G$ is of the form

$$
G(t, y)=H\left(\frac{y}{v(t)}\right) \frac{\left|v^{\prime}(t)\right|}{v(t)}
$$

with some monotone nonincreasing continuous function $H:(a, \infty] \rightarrow \mathbb{R}, H \geq 0$. Assume that $\int_{a}^{\infty} H(y) \mathrm{d} y>0$, and that $v$ is not constant in $(1-\varepsilon, 1)$ for some $\varepsilon>0$, so that 1 is the critical point for $c$. Define $v(1):=\lim _{t \uparrow 1} v(t) \in \overline{\mathbb{R}}$. The following example shows how the case of separate variables fits into the form given in (3.3).

Example 3.1. Let $a:[0,1] \rightarrow[0, \infty]$ be continuous and integrable, and define $A(t):=$ $\int_{t}^{1} a(s) \mathrm{d} s$.

(a) For $\alpha>1$, the intensity function $G(t, y)=a(t) y^{-\alpha}$ on $[0,1) \times(0, \infty]$ is of the form given in (3.3) with $H(y)=(\alpha-1) y^{-\alpha}$ and $v(t)=((\alpha /(\alpha-1)) A(t))^{1 / \alpha}$.

(b) For $\alpha>0$, the intensity function $G(t, y)=a(t)(-y)^{\alpha}$ for $y \leq 0$ and $G(t, y)=0$ for $y>0$ on $[0,1) \times(-\infty, \infty]$ is of the form given in (3.3) with $H(y)=0$ for $y>0$ and $H(y)=(\alpha+1)(-y)^{\alpha}$ for $y \leq 0$, and $v(t)=((\alpha /(\alpha+1)) A(t))^{-1 / \alpha}$. 
Representation (3.3) is in general even for fixed $H$ not unique since $v$ has to satisfy only a differential equation without an initial value. It depends on $H$, for which initial value solutions can be found. We distinguish three cases: $v(1)=0, v(1)=1$, and $v(1)=\infty$. (If $v(1)=d \in$ $(0, \infty)$, consider $\tilde{v}(t):=v(t) / d$ and absorb the $d$ into $H$ by considering $\tilde{H}(x):=H(x / d)$.) The cases $v(1)=0$ and $v(1)=\infty$ lead to a simpler structure of solutions.

For $v$ monotonically nonincreasing, we define

$$
R(x):=x-\int_{x}^{\infty} H(y) \mathrm{d} y, \quad x \in[a, \infty) .
$$

Then $R:[a, \infty) \rightarrow \overline{\mathbb{R}}$ is concave and monotonically nondecreasing.

For $v$ monotonically nondecreasing, we define

$$
R(x):=x+\int_{x}^{\infty} H(y) \mathrm{d} y, \quad x \in(a, \infty), \quad R(a):=\lim _{x \downarrow a} R(x) .
$$

In this case $R:[a, \infty) \rightarrow \overline{\mathbb{R}}$ is convex.

The form of solutions of the optimality equation (2.3) with boundary value $x$ depends critically on the existence and on the number of zero points of $R$. In the following we reduce the problem of solving the optimality equation (2.3) for all boundary values $x$ to finding solutions $\Phi$ of the differential equation

$$
\Phi^{\prime}(x)=\frac{\Phi(x)}{R(x)} \neq 0 .
$$

Solutions of (3.6) are given, e.g. by

$$
\Phi(x)=\exp \left(\int_{x_{0}}^{x} \frac{1}{R(y)} \mathrm{d} y\right)
$$

with $x_{0}$ chosen such that the integral exists. The inverse mapping $\phi$ of $\Phi$ exists and solves the equation

$$
\phi^{\prime}(z)=\frac{R(\phi(z))}{z} .
$$

The definitions of $R$ given in (3.4) and (3.5), and the solutions of (3.6) and (3.7) will allow us in the following to construct solutions of the optimality equation (2.3) for any boundary values and to verify the separation condition (S).

In the following we omit some of the simple calculations. We distinguish four cases.

Case 1: $v$ monotonically nonincreasing, $v(1)=0$. Then $c=0$. Let $a \geq 0$, and assume that $R(r)=0$ for some $r>a$. Then the separation condition $(S)$ is satisfied and the optimal stopping curves are given by

$$
u(t, x)=\phi\left(\frac{x}{v(t)}\right) v(t), \quad(t, x) \in[0,1) \times[0, \infty),
$$

where $\phi:[0, \infty) \rightarrow[r, \infty)$ is the inverse to

$$
\Phi:[r, \infty) \rightarrow[0, \infty), \quad \Phi(x)=x \exp \left(-\int_{x}^{\infty}\left(\frac{1}{R(y)}-\frac{1}{y}\right) \mathrm{d} y\right) .
$$

In particular, the optimal solution $u$ is given explicitly as $u(t)=r v(t)$. 
For the proof, we first note that $\Phi$ satisfies the differential equation (3.6) and $\phi$ satisfies (3.7), and then establish that $u$ satisfies the optimality equation (2.3). By definition,

$$
u(t, x)=\phi\left(\frac{x}{v(t)}\right) \frac{v(t)}{x} x \rightarrow x \quad \text { as } t \rightarrow 1,
$$

since $\lim _{y \rightarrow \infty} \phi(y) / y=\lim _{x \rightarrow \infty} x / \Phi(x)=1$. Thus, the boundary condition is satisfied. Furthermore,

$$
\begin{aligned}
\frac{\partial}{\partial t} u(t, x) & =\phi^{\prime}\left(\frac{x}{v(t)}\right) \frac{-x v^{\prime}(t)}{v(t)^{2}} v(t)+\phi\left(\frac{x}{v(t)}\right) v^{\prime}(t) \\
& =\frac{R(\phi(x / v(t)))}{x / v(t)} \frac{-x v^{\prime}(t)}{v(t)}+\phi\left(\frac{x}{v(t)}\right) v^{\prime}(t) \\
& =\left(-\phi\left(\frac{x}{v(t)}\right)+\int_{\phi(x / v(t))}^{\infty} H(y) \mathrm{d} y\right) v^{\prime}(t)+\phi\left(\frac{x}{v(t)}\right) v^{\prime}(t) \\
& =\int_{\phi(x / v(t)) v(t)}^{\infty} H\left(\frac{y}{v(t)}\right) \frac{v^{\prime}(t)}{v(t)} \mathrm{d} y \\
& =-\int_{u(t, x)}^{\infty} G(t, y) \mathrm{d} y .
\end{aligned}
$$

Thus, $u(\cdot, x)$ solves the optimality equation $(2.3)$.

It remains to show that $\int_{x}^{\infty}(1 / R(y)-1 / y) \mathrm{d} y<\infty$ for $x \in(r, \infty)$. With $I(y):=$ $\int_{y}^{\infty} H(x) \mathrm{d} x$ we have

$$
\frac{1}{R(y)}-\frac{1}{y}=\frac{I(y)}{y(y-I(y))}=\frac{1}{y^{2}} \frac{I(y)}{1-I(y) / y} \leq C \frac{1}{y^{2}},
$$

and, thus, the integral is finite.

Case 2: $v$ monotonically nondecreasing, $v(1)=\infty$. In this case we have $c=-\infty$. Let $a<0$, and assume that $R(r)=0$ for some $a<r<0$. We also assume in this case that

$$
\int_{0}^{\infty} H(x) \mathrm{d} x=0 \text { and } \int_{y}^{0} \frac{H(x)}{-x} \mathrm{~d} x<\infty \text { for } y<0 .
$$

Under this assumption, the separation condition (S) is satisfied and the optimal stopping curves are given, for $(t, x) \in[0,1) \times \overline{\mathbb{R}}$, by

$$
u(t, x)= \begin{cases}x & \text { if } x \geq 0 \\ \phi\left(\frac{x}{v(t)}\right) v(t) & \text { if } x<0\end{cases}
$$

with $\phi:[-\infty, 0] \rightarrow[r, 0]$ the inverse of

$$
\Phi:[r, 0] \rightarrow[-\infty, 0], \quad \Phi(x):=x \exp \left(\int_{x}^{0}\left(\frac{1}{y}-\frac{1}{R(y)}\right) \mathrm{d} y\right) .
$$

In particular, the optimal solution $u$ is given by $u(t)=r v(t)$. 
For the proof, note that $\Phi$ solves the differential equation (3.6), while the inverse $\phi$ solves (3.7). We have to establish that $\int_{x}^{0}(1 / y-1 / R(y)) \mathrm{d} y<\infty$ for $x \in(r, 0)$. Again, with $I(y):=\int_{y}^{0} H(x) \mathrm{d} x$ we obtain the estimate

$$
\frac{1}{y}-\frac{1}{R(y)}=\frac{I(y)}{y(y+I(y))}=\frac{1}{-y} \frac{I(y) /(-y)}{1-I(y) /(-y)} \leq \frac{1}{-y} \frac{H(y)}{1-H(y)} \leq C \frac{H(y)}{-y}
$$

for $y<0$ with $H(y) \leq 1-1 / C<1$. By assumption, this is integrable. As in case 1 , we find, by similar calculations, that $u(t, x)$ satisfies the optimality equation (2.3). Thus, the result follows.

The following two cases are derived similarly to cases 1 and 2, and, therefore, we only state the results.

Case 3: $v$ monotonically nonincreasing, $v(1)=1$. Then $c=a$. Let $r>c$ be such that $R(r)=0$. We assume that $\int_{c}^{x} R(y)^{-1} \mathrm{~d} y>-\infty$ for some $x \in(c, r)$. This is, e.g. the case when $c \in \mathbb{R}$. Under this assumption, the separation condition (S) is satisfied and the optimal stopping curves are given, for $(t, x) \in[0,1) \times[c, \infty)$, by

$$
u(t, x)= \begin{cases}\phi_{1}\left(\frac{\Phi_{1}(x)}{v(t)}\right) v(t) & \text { if } r<x<\infty, \\ x v(t) & \text { if } x=r, \\ \phi_{2}\left(\frac{\Phi_{2}(x)}{v(t)}\right) v(t) & \text { if } c \leq x<r,\end{cases}
$$

where $\Phi_{1}:(r, \infty) \rightarrow \mathbb{R}$ and $\Phi_{2}:[c, r) \rightarrow \mathbb{R}$ are solutions of (3.6), and $\phi_{1}$ and $\phi_{2}$ are the inverses. The function $\Phi_{2}$ can be chosen as $\Phi_{2}(x):=\exp \left(\int_{c}^{x} R(y)^{-1} \mathrm{~d} y\right)$.

The boundary case $x=r$ is particularly simple here.

Case 4: $v$ monotonically nondecreasing, $v(1)=1$. Then $c=a$. We have to distinguish three cases. In each of these cases the solution is similar to case 3 and will therefore be omitted.

In conclusion, the $v(1)=0$ and $v(1)=\infty$ cases yield solutions of a simpler structure compared to the $v(1)=1$ case. Thus, a representation of $G$ as in (3.3) — which is not uniqueis preferable if it leads to one of the first two cases.

\subsection{Second class of intensity functions}

Let $f=a+v$ on $[0,1)$ with $a \in \mathbb{R} \cup\{-\infty\}$ and a monotone $C^{1}$-function $v:[0,1) \rightarrow \mathbb{R}$ with $v(1):=\lim _{t \uparrow 1} v(t)$.

We consider intensity functions on $M_{f} \cap[0,1) \times \overline{\mathbb{R}}$ of the form

$$
G(t, y)=H(y-v(t))\left|v^{\prime}(t)\right|
$$

with a continuous monotonically nonincreasing function $H:(a, \infty] \rightarrow \mathbb{R}, H \geq 0$, such that $\int_{a}^{\infty} H(y) \mathrm{d} y>0$. We assume that $v$ is not constant in $(1-\varepsilon, 1)$ for some $\varepsilon>0$, so that 1 is the critical point of $c$.

As an example, let $a:[0,1] \rightarrow[0, \infty]$ be continuous and integrable, and let, as in Example 3.1, $A(t):=\int_{t}^{1} a(s) \mathrm{d} s$. Then the intensity function $G(t, y)=a(t) \mathrm{e}^{-y}$ on $[0,1) \times$ $(-\infty, \infty]$ is a case of separate variables. It fits into the form (3.8) with $H(y)=\mathrm{e}^{-y}$ and $v(t)=\log A(t)$.

If $v$ is monotonically nonincreasing then we define

$$
R(x):=1-\int_{x}^{\infty} H(y) \mathrm{d} y \text { for } x \in[a, \infty)
$$


The function $R:[a, \infty) \rightarrow[-\infty, 1)$ is concave and monotonically nondecreasing. If $v$ is monotonically nondecreasing then we define

$$
R(x):=1+\int_{x}^{\infty} H(y) \mathrm{d} y \text { for } x \in[a, \infty) .
$$

In this case $R:[a, \infty) \rightarrow[1, \infty)$ is convex and monotonically nonincreasing.

We construct optimal stopping curves by means of solutions of the equation

$$
\Phi^{\prime}(x)=\frac{1}{R(x)} .
$$

Equation (3.9) is solved, e.g. by $\Phi(x)=\int_{x_{0}}^{x}(1 / R(y)) \mathrm{d} y$, where $x_{0}$ is chosen such that the integral exists. The inverse function $\phi$ of $\Phi$ satisfies

$$
\phi^{\prime}(z)=R(\phi(z))
$$

Similarly to the examples in cases $1-4$, we obtain explicit forms for the solution $u(t, x)$ of the optimal stopping curves. The arguments are similar and, therefore, we essentially only state the results.

Case $(i): v$ monotonically nonincreasing, $v(1)=-\infty$. Then $c=-\infty$. Assume that $R(r)=0$ for some $r>a$. We further assume here that

$$
\int_{z}^{\infty} \int_{y}^{\infty} H(x) \mathrm{d} x \mathrm{~d} y<\infty \text { for } z>r .
$$

Then the separation condition (S) is satisfied and the optimal stopping curves for $(t, x) \in$ $[0,1) \times \overline{\mathbb{R}}$ are given by

$$
u(t, x)=\phi(x-v(t))+v(t)
$$

where $\phi: \overline{\mathbb{R}} \rightarrow[r, \infty]$ is the inverse of $\Phi:[r, \infty] \rightarrow \overline{\mathbb{R}}$, given by

$$
\Phi(x):=x-\int_{x}^{\infty}\left(\frac{1}{R(y)}-1\right) \mathrm{d} y .
$$

The optimal stopping curve $u$ is given by $u(t)=r+v(t)$.

For the proof, note that $\Phi$ solves the differential equation $\Phi^{\prime}(x)=1 / R(x)$ and $\phi$ solves $\phi^{\prime}(z)=R(\phi(z))$. Consequently, as in case 1 , we find that $u(\cdot, x)$ solves the optimality equation (2.3) with boundary value $x$. We still need to show that $\int_{x}^{\infty}(1 / R(y)-1) \mathrm{d} y<\infty$ for $x \in$ $(r, \infty)$. With $I(y):=\int_{y}^{\infty} H(x) \mathrm{d} x$ we obtain the bound

$$
\frac{1}{R(y)}-1=\frac{I(y)}{1-I(y)} \leq C I(y) .
$$

The last term is integrable by assumption.

The next two cases allow similar explicit solutions but are not used in the applications in Section 5 and, therefore, are not explicitly stated. For details, see Faller (2009).

Case(ii): $v$ monotonically decreasing, $v(1)=0$.

Case(iii): $v$ monotonically increasing, $v(1)=0$. 
Remark 3.1. We can extend the class of intensity functions for which solutions can be given in a simple way by translations. Let $N$ satisfy conditions (B), (S), and (D) with intensity function $G$ on $M_{f}$. For $d \in \mathbb{R}$, consider the intensity function

$$
G_{d}(t, y):=G(t, y-d), \quad(t, y) \in M_{f+d} .
$$

Then the optimal stopping curves $u_{d}(\cdot, x)$ with respect to $G_{d}$ and $c_{d}:=c+d$ are given by

$$
u_{d}(t, x)=u(t, x-d)+d \quad \text { for }(t, x) \in[0,1] \times\left[c_{d}, \infty\right]
$$

For $x \in \mathbb{R},(3.10)$ follows by a simple calculation. For $x=-\infty,(3.10)$ follows by means of Lemma 2.2.

As an application of Remark 3.1, we consider the following example, which is relevant in Section 5 for the stopping of i.i.d. sequences with discount and observation costs.

Example 3.2. In this example $c \in \mathbb{R}$ denotes a real constant and the guarantee value is $-\infty$. On $[0,1) \times \overline{\mathbb{R}}$, consider

$$
G_{c, d}(t, y)= \begin{cases}0 & \text { if } \frac{y}{v(t)} \geq d, \\ \frac{1}{t}\left(-\frac{y}{v(t)}+d\right)^{\alpha} & \text { if } \frac{y}{v(t)}<d,\end{cases}
$$

with $v(t):=t^{c-1 / \alpha}$, where $\alpha>0$ and $c, d \in \mathbb{R}$ with $c \neq 1 / \alpha$. These intensity functions satisfy (3.3) with

$$
H(x):= \begin{cases}0 & \text { if } x \geq d, \\ \frac{\alpha}{|1-c \alpha|}(-x+d)^{\alpha} & \text { if } x<d .\end{cases}
$$

By cases 3 and 4, the optimal stopping curve $u_{c, d}$ of the Poisson process $N=N_{c, d}$ with intensity function $G=G_{c, d}$, where $u_{c, d}(t):=u_{c, d}(t,-\infty)$, is given by

$$
u_{c, d}(t)=\phi_{c, d}\left(\frac{1}{v(t)}\right) v(t)
$$

Here $\phi_{c, d}$ is the inverse of

$$
\Phi_{c, d}(x):=\exp \left(\int_{-\infty}^{x} \frac{1}{R_{c, d}(y)} \mathrm{d} y\right) .
$$

The function $\Phi_{c, d}$ is defined on $[-\infty, r]$, where $r$ is the smallest zero point of

$$
R_{c, d}(x):= \begin{cases}x & \text { if } x \geq d, \\ x-\frac{\alpha}{\alpha+1} \frac{1}{1-c \alpha}(-x+d)^{\alpha+1} & \text { if } x<d,\end{cases}
$$

or $r:=\infty$ if no zeros exist. The function $\phi_{c, d}$ is defined on $[0,1]$ if $c<1 / \alpha$, and on $[1, \infty]$ if $c>1 / \alpha$. 
For $d=0$, all functions can be calculated explicitly. The primitive of $1 /\left(y-c(-y)^{\alpha+1}\right)$ is given by $-(1 / \alpha) \log \left|(-y)^{-\alpha} / c+1\right|$ and, consequently, we obtain

$$
\begin{aligned}
u_{c, 0}(t) & =-\left(\frac{\alpha}{\alpha+1} \frac{1}{1-c \alpha}\left(1-t^{1-c \alpha}\right)\right)^{-1 / \alpha}, \\
u_{c, 0}(t, x) & = \begin{cases}x & \text { if } x \geq 0, \\
-\left(\frac{\alpha}{\alpha+1} \frac{1}{1-c \alpha}\left(1-t^{1-c \alpha}\right)+(-x)^{-\alpha}\right)^{-1 / \alpha} & \text { if } x<0 .\end{cases}
\end{aligned}
$$

For $d \neq 0$ and general $\alpha, \Phi_{c, d}$ and $\phi_{c, d}$ cannot be calculated explicitly. We can, however, derive the following bounds (see Faller (2009) for details).

If $c>1 / \alpha$ and $d>0$ or $c<1 / \alpha$ and $d<0$, then, for all $(t, x) \in[0,1] \times \overline{\mathbb{R}}$,

$$
u_{c, 0}(t, x-d v(t))+d v(t) \leq u_{c, d}(t, x) \leq u_{c, 0}(t, x-d)+d .
$$

In the other cases $c>1 / \alpha$ and $d<0$ or $c<1 / \alpha$ and $d>0$,

$$
u_{c, 0}(t, x-d)+d \leq u_{c, d}(t, x) \leq u_{c, 0}(t, x-d v(t))+d v(t) .
$$

In particular, we obtain in all cases

$$
\lim _{t \uparrow 1}\left(u_{c, d}(t)-u_{c, 0}(t)\right)=d .
$$

Equation (3.14) opens up another way of calculating $u_{c, d}$ numerically. In the first step we should solve the differential equation for $u_{c, d}-u_{c, 0}$. This is relieved by the fact that the initial value $d$ is finite. In the second step we just add the explicitly known constant $u_{c, 0}$ to obtain $u_{c, d}$.

For the proof of (3.13), assume that $d<0$ and $c<1 / \alpha$, and, thus, that $v$ is monotonically nonincreasing. The other cases follow similarly. Choose $t_{1} \in[0,1)$. Then, for $t \in\left[t_{1}, 1\right)$, $\varepsilon:=v\left(t_{1}\right) \geq v(t)>1$ and we have

$$
G_{c, d}(t, y) \geq G_{c, 0}(t, y-\varepsilon d) .
$$

By the comparison result (see Proposition 2.3) and Remark 3.1, we obtain, for the optimal stopping curves,

$$
u_{c, d}(t, x) \geq u_{c, 0}(t, x-\varepsilon d)+\varepsilon d \quad \text { for } t \geq t_{1} .
$$

This holds in particular for $t=t_{1}$. In the opposite direction we have, for all $t$,

$$
G_{c, d}(t, y) \leq G_{c, 0}(t, y-d)
$$

and, thus,

$$
u_{c, d}(t, x) \leq u_{c, 0}(t, x-d)+d
$$

\section{Approximation of optimal stopping problems}

In this section we state an extension of the approximation result in Kühne and Rüschendorf (2004, Theorem 2.1) for optimal stopping problems for dependent sequences. In particular, we add essential information to the important case $c=-\infty$. We also extend the approximation 
result to general filtrations, which is useful when dealing with dependent sequences. In Section 5 we apply this extended approximation result and the developments of the previous sections to the optimal stopping of i.i.d. sequences with discount and observation costs.

As in Section 2, let $N$ be a Poisson process on $M_{f}$, let the intensity measure $\mu$ have Lebesgue density $g$, and let $c:=f(1)$ and $t_{0}(c)=1$. Let $u(t, x)$ and $u(t)$ denote the optimal stopping curves of $N$, let $T(t, x)$ and $\bar{Y}_{T(t, x)}$ denote the optimal stopping times and rewards, and assume that conditions (B) and (S) hold in general.

For $n \in \mathbb{N}$, let $X_{1}^{n}, \ldots, X_{n}^{n}$ be real random variables with $\mathrm{E}\left(X_{i}^{n}\right)^{+}<\infty$ adapted to the filtration $\mathcal{F}^{n}=\left(\mathcal{F}_{i}^{n}\right)_{0 \leq i \leq n}$ and such that $\mathcal{F}_{\lfloor t n\rfloor}^{n} \subset \mathcal{F}_{\lfloor t(n+1)\rfloor}^{n+1}$. For the imbedded point process,

$$
N_{n}:=\sum_{i=1}^{n} \delta_{\left(i / n, X_{i}^{n}\right)}
$$

in $[0,1] \times \overline{\mathbb{R}}$, we define the optimal stopping curve with respect to $\mathcal{F}^{n}$ with guarantee value $x \in[c, \infty]$ by

$$
\begin{aligned}
u_{n}(t, x) & :=W_{\mathcal{F}^{n}}\left(X_{\lfloor t n\rfloor+1}^{n} \vee x, \ldots, X_{n}^{n} \vee x\right), \quad t \in[0,1), \\
u_{n}(1, x) & :=x
\end{aligned}
$$

Here $W_{\mathcal{F}^{n}}$ denotes the optimal stopping value over all $\mathcal{F}^{n}$-stopping times. In detail, (4.1) is given by

$$
\begin{aligned}
u_{n}(t, x) & =\operatorname{esssup}\left\{\mathrm{E}\left[X_{T}^{n} \vee x \mid \mathcal{F}_{\lfloor t n\rfloor}^{n}\right]: T>t n, T \text { is an } \mathcal{F}^{n} \text {-stopping time }\right\} \\
& =\mathrm{E}\left[X_{T_{n}(t, x)}^{n} \vee x \mid \mathcal{F}_{\lfloor t n\rfloor}^{n}\right] \quad \mathrm{P} \text {-a.s., }
\end{aligned}
$$

with optimal stopping times

$$
T_{n}(t, x):=\min \left\{t n<i \leq n: X_{i}^{n}>u_{n}\left(\frac{i}{n}, x\right)\right\}, \quad T_{n}(1, x):=n .
$$

The function $u_{n}(\cdot, x)$ is a right-continuous piecewise constant curve. It is monotone in the sense that, for $0 \leq s \leq t \leq 1$,

$$
u_{n}(s, x) \geq \mathrm{E}\left[u_{n}(t, x) \mid \mathcal{F}_{\lfloor s n\rfloor}^{n}\right] \quad \mathrm{P} \text {-a.s. }
$$

In the other direction, inductively by the recursive definition of optimal thresholds, for $0 \leq s \leq$ $t \leq 1$, we obtain

$$
u_{n}(s, x) \leq \mathrm{E}\left[\max _{s<i / n \leq t} X_{i}^{n} \vee u_{n}(t, x) \mid \mathcal{F}_{\lfloor s n\rfloor}^{n}\right] \quad \mathrm{P} \text {-a.s. }
$$

An important condition in the dependent case is the following asymptotic independence condition.

(A) For $0 \leq s<t \leq 1$,

$$
\mathrm{P}\left(\max _{s<i / n \leq t} X_{i}^{n} \vee f(s) \leq x \mid \mathcal{F}_{\lfloor s n\rfloor}^{n}\right) \stackrel{\mathrm{P}}{\rightarrow} \mathrm{P}\left(\sup _{s<\tau_{k} \leq t} Y_{k} \vee f(s) \leq x\right) \quad \text { for all } x \in \mathbb{R} .
$$


We need the uniform integrability condition:

(U) $M_{n}^{+}$, with $M_{n}:=\max _{1 \leq i \leq n} X_{i}^{n}$, is uniformly integrable and $\mathrm{E}\left[\lim \sup _{n \rightarrow \infty} M_{n}^{+}\right]<\infty$.

The addition $\mathrm{E}\left[\lim \sup _{n} M_{n}^{+}\right]<\infty$ can be omitted when $\mathcal{F}^{n}$ is the canonical filtration and $N_{n} \stackrel{\mathrm{D}}{\rightarrow} N$ on $([0,1] \times \overline{\mathbb{R}}) \backslash \operatorname{graph}(f)$. In this case the Skorokhod theorem is applicable and the above additional condition is a consequence of condition (B) for $N$, which is assumed throughout this paper. To ensure uniform integrability in the $c=-\infty$ case, the following uniform integrability condition from below is postulated, which is a functional version of the corresponding condition in Kühne and Rüschendorf (2004).

(L) For some sequence $\left(v_{n}\right)_{n \in \mathbb{N}}$ of monotonically nonincreasing functions $v_{n}:[0,1] \rightarrow$ $\mathbb{R} \cup\{-\infty\}$ with $v_{n} \rightarrow u$ pointwise, all $t \in[0,1)$, and the corresponding threshold stopping times

$$
\hat{T}_{n}(t):=\min \left\{t n<i \leq n: X_{i}^{n}>v_{n}\left(\frac{i}{n}\right)\right\}
$$

it holds that

$$
\lim _{s \uparrow 1} \limsup _{n \rightarrow \infty} \mathrm{E}\left[X_{\hat{T}_{n}(t)}^{n} \chi_{\left\{\hat{T}_{n}(t)>s n\right\}}\right]=0 .
$$

Conditions (L) and (U) imply uniform integrability of $\left(X_{\hat{T}_{n}(t)}^{n}\right)_{n \in \mathbb{N}}($ see Faller (2009, p. 30)). For notational convenience, we write

$$
T_{n}:=T_{n}(0, c) \quad \text { and } \quad T:=T(0, c) .
$$

Theorem 4.1. (Approximation of stopping problems.) Assume that $N_{n} \stackrel{\mathrm{D}}{\rightarrow} N$ on $M_{f}$, and that conditions $(A)$ and $(U)$ hold. If $c=-\infty$ then we additionally assume that condition $(L)$ holds.

(a) For all $(t, x) \in[0,1] \times[c, \infty)$,

$$
u_{n}(t, x) \stackrel{\mathrm{P}}{\rightarrow} u(t, x) .
$$

If $c \in \mathbb{R}$ and assuming that $\mu\left(M_{u}\right)=\infty$ or $X_{n}^{n} \stackrel{\mathrm{P}}{\rightarrow} c$, then

$$
\left(\frac{T_{n}}{n}, X_{T_{n}}^{n}\right) \stackrel{\mathrm{D}}{\rightarrow}\left(T, \bar{Y}_{T} \vee c\right) .
$$

(b) If $c \in \mathbb{R}$ and $X_{n}^{n} \stackrel{L^{1}}{\rightarrow} c$, then $\hat{T}_{n}:=\min \left\{1 \leq i \leq n: X_{i}^{n}>u(i / n)\right\}$ is an asymptotically optimal sequence of stopping times, i.e.

$$
\mathrm{E} X_{\hat{T}_{n}}^{n} \rightarrow u(0)
$$

If $c=-\infty$ then $\hat{T}_{n}:=\min \left\{1 \leq i \leq n: X_{i}^{n}>v_{n}(i / n)\right\}$, with $v_{n}$ from condition $(L)$, is an asymptotically optimal sequence of stopping times and $\mathrm{E} X_{\hat{T}_{n}}^{n} \rightarrow u(0)$.

For a detailed proof of this extended approximation result, we refer the reader to Faller (2009, Satz 1.20). 


\section{Optimal stopping of i.i.d. sequences with discount and observation costs}

Based on the results in Sections 2-4, we are able to give a fairly complete treatment of the optimal stopping problem of i.i.d. sequences with discount and observation costs. Some particular instances of this problem were established in Kühne and Rüschendorf (2000b). The problem goes back to Kennedy and Kertz (1990), (1991) in the i.i.d. case.

Let $\left(Z_{i}\right)$ be an i.i.d. sequence with distribution function $F$ in the domain of attraction of an extreme value distribution $G$; thus, for some constants $a_{n}>0, b_{n} \in \mathbb{R}$,

$$
n\left(1-F\left(a_{n} x+b_{n}\right)\right) \rightarrow-\log G(x), \quad x \in \mathbb{R} .
$$

Consider $X_{i}=c_{i} Z_{i}+d_{i}$, the sequence with discount and observation factors, $c_{i}>0, d_{i} \in \mathbb{R}$, and both sequences monotonically nondecreasing or nonincreasing. For convergence of the corresponding imbedded point processes,

$$
\hat{N}_{n}=\sum_{i=1}^{n} \delta_{\left(i / n,\left(X_{i}-\hat{b}_{n}\right) / \hat{a}_{n}\right)},
$$

the following choices of $\hat{a}_{n}$ and $\hat{b}_{n}$ turn out to be appropriate:

$$
\begin{gathered}
\hat{a}_{n}:=c_{n} a_{n}, \quad \hat{b}_{n}:=0, \quad \text { for } F \in D\left(\Phi_{\alpha}\right) \text { or } F \in D\left(\Psi_{\alpha}\right), \\
\hat{a}_{n}:=c_{n} a_{n}, \quad \hat{b}_{n}:=c_{n} b_{n}+d_{n} \quad \text { for } F \in D(\Lambda) .
\end{gathered}
$$

Here $\Phi_{\alpha}, \Psi_{\alpha}$, and $\Lambda$ are the Fréchet, Weibull, and Gumbel distributions, and $a_{n}$ and $b_{n}$ are the corresponding normalizations in (5.1). We give further conditions on $c_{i}$ and $d_{i}$ to establish point process convergence in (5.2). Related conditions are given in De Haan and Verkaade (1987) and in Kühne and Rüschendorf (2000b) in the treatment of i.i.d. sequences with trends.

In the following $c$ denotes some general constant and not as before the guarantee value. The guarantee value of $N$ is given by 0 in the $\Phi_{\alpha}$ case and generally by $-\infty$ in the $\Psi_{\alpha}$ and $\Lambda$ cases. This application shows the importance of treating the case with lower boundary $-\infty$ as in Sections 2-4. We state the optimality results for all three cases.

Theorem 5.1. Let $F \in D\left(\Phi_{\alpha}\right)$ with $\alpha>1$ and $F(0)=0$. Also, let $b_{n}=0$, and assume that

$$
\frac{d_{n}}{c_{n} a_{n}} \rightarrow d, \quad \frac{c_{\lfloor t n\rfloor}}{c_{n}} \rightarrow t^{c} \quad \text { for all } t \in[0,1]
$$

for constants $c, d \in \mathbb{R}$, and that $c_{n}$ does not converge to 0 . Then

$$
\frac{\mathrm{E} X_{T_{n}}}{\hat{a}_{n}} \rightarrow \begin{cases}\infty & \text { if } c \leq-\frac{1}{\alpha}, \\ d \exp \left(\int_{d}^{\infty}\left(\frac{1}{x}-\frac{1}{R(x)}\right) \mathrm{d} x\right) & \text { if } c>-\frac{1}{\alpha}, d>0, \\ \left(\frac{\alpha}{\alpha-1} \frac{1}{1+c \alpha}\right)^{1 / \alpha} & \text { if } c>-\frac{1}{\alpha}, d=0, \\ \exp \left(\int_{1}^{\infty}\left(\frac{1}{x}-\frac{1}{R(x)}\right) \mathrm{d} x-\int_{d}^{1} \frac{1}{R(x)} \mathrm{d} x\right) & \text { if } c>-\frac{1}{\alpha}, d<0, r=\infty, \\ 0 & \text { if } c>-\frac{1}{\alpha}, d<0, r<\infty,\end{cases}
$$


where $r>d$ is the smallest zero point of

$$
R(x):=x+\frac{\alpha}{\alpha-1} \frac{1}{1+c \alpha}(x-d)^{-\alpha+1}, \quad x \in(d, \infty),
$$

and $r:=\infty$ if $R$ has no zero point greater than $d$.

For the values of $c$ and $d$ where the limit in (5.3) is not 0 or $\infty$, we determine asymptotically optimal sequences of stopping times. For $c>-1 / \alpha$, define

$$
u(t):=\phi\left(\frac{1}{v(t)}\right) v(t)
$$

with $v(t):=t^{c+1 / a}$ and $\phi$ the inverse of $\Phi:[d, r] \rightarrow[1, \infty]$, given by

$$
\Phi(x):=\exp \left(\int_{d}^{x} \frac{1}{R(y)} \mathrm{d} y\right) .
$$

Then $\hat{T}_{n}:=\min \left\{1 \leq i \leq n: X_{i}>\hat{a}_{n} u(i / n)\right\}$ is an asymptotically optimal sequence of stopping times, i.e. the sequence of normalized expectations has the same limit as in (5.3).

Theorem 5.2. Let $F \in D\left(\Psi_{\alpha}\right)$ with $\alpha>0$ and $F(0)=1$. Also, let $a_{n} \downarrow 0$ and $b_{n}=0$, and assume that

$$
\frac{d_{n}}{c_{n} a_{n}} \rightarrow d, \quad \frac{c_{\lfloor t n\rfloor}}{c_{n}} \rightarrow t^{c} \quad \text { for all } t \in[0,1]
$$

for constants $c, d \in \mathbb{R}$. If $d_{n}>0$ then assume that either $\left(d_{n}\right)_{n \in \mathbb{N}}$ is monotonically nondecreasing or $c_{n} a_{n}$ does not converge to 0 . Then

$$
\frac{\mathrm{E} X_{T_{n}}}{\hat{a}_{n}} \rightarrow \begin{cases}\infty & \text { if } c<\frac{1}{\alpha}, d>0, \\ -\left(\frac{\alpha}{\alpha+1} \frac{1}{1-c \alpha}\right)^{-1 / \alpha} & \text { if } c<\frac{1}{\alpha}, d=0, \\ \exp \left(-\int_{-\infty}^{d} \frac{1}{R(x)} \mathrm{d} x\right) & \text { if } c<\frac{1}{\alpha}, d<0, \\ 0 & \text { if } c=\frac{1}{\alpha}, \\ d \exp \left(-\int_{-\infty}^{d} \frac{1}{R(x)} \mathrm{d} x\right) & \text { if } c>\frac{1}{\alpha}, r=\infty(\Rightarrow d>0), \\ 0 & \text { if } c>\frac{1}{\alpha}, r<\infty,\end{cases}
$$

where $r$ is the smallest zero point of

$$
R(x):= \begin{cases}x & \text { if } x \geq d, \\ x-\frac{\alpha}{\alpha+1} \frac{1}{1-c \alpha}(-x+d)^{\alpha+1} & \text { if } x<d,\end{cases}
$$

or $r:=\infty$ if $R$ has no zero point. 
For the values of $c$ and $d$ where the limit of (5.5) is not 0 or $\infty$, we can construct asymptotically optimal sequences of stopping times. Let $\left(w_{n}\right)_{n \in \mathbb{N}}$ be a monotonically nondecreasing sequence of negative constants with

$$
\lim _{n \rightarrow \infty} n\left(1-F\left(w_{n}\right)\right)=\frac{\alpha+1}{\alpha},
$$

as, e.g. $w_{n}:=-((\alpha+1) / \alpha)^{1 / \alpha} a_{n}$. For $c \neq 1 / \alpha$, let $u_{c, d}$ be the solutions derived in (3.12) and define

$$
v_{n}(t):=\frac{u_{c, 0}(t)}{u_{0,0}(t)} \frac{w_{\lfloor(1-t) n\rfloor}}{a_{n}}+u_{c, d}(t)-u_{c, 0}(t) \quad \text { for } t \in[0,1), \quad v_{n}(1):=-\infty,
$$

where

$$
u_{c, 0}(t)=-\left(\frac{\alpha}{\alpha+1} \frac{1}{1-c \alpha}\left(1-t^{1-c \alpha}\right)\right)^{-1 / \alpha}
$$

and

$$
u_{0,0}(t)=-\left(\frac{\alpha}{\alpha+1}(1-t)\right)^{-1 / \alpha}
$$

Then $\hat{T}_{n}:=\min \left\{1 \leq i \leq n: X_{i}>\hat{a}_{n} v_{n}(i / n)\right\}$ is an asymptotically optimal sequence of stopping times.

Theorem 5.3. Let $F \in D(\Lambda)$, and assume that

$$
\frac{b_{n}}{a_{n}}\left(1-\frac{c_{\lfloor t n\rfloor}}{c_{n}}\right) \rightarrow c \log (t), \quad \frac{d_{n}-d_{\lfloor t n\rfloor}}{c_{n} a_{n}} \rightarrow d \log (t) \quad \text { for all } t \in[0,1]
$$

for constants $c, d \in \mathbb{R}$. Assume that $\left(c_{n}\right)_{n \in \mathbb{N}}$ and $\left(d_{n}\right)_{n \in \mathbb{N}}$ are monotonically nondecreasing. Then

$$
\frac{\mathrm{E} X_{T_{n}}-\hat{b}_{n}}{\hat{a}_{n}} \rightarrow \begin{cases}\infty & \text { if } c+d \geq 1 \\ \log \left(\frac{1}{1-(c+d)}\right) & \text { if } c+d<1 .\end{cases}
$$

For $c+d<1$, let $\left(w_{n}\right)_{n \in \mathbb{N}}$ be monotonically nondecreasing with $\lim _{n \rightarrow \infty} n\left(1-F\left(w_{n}\right)\right)=1$, as, e.g. $w_{n}:=b_{n}$. Let

$$
u(t):=\log \left(\frac{1}{1-(c+d)}\left(1-t^{1-(c+d)}\right)\right)
$$

and

$$
v_{n}(t):=\frac{w_{\lfloor(1-t) n\rfloor}-b_{n}}{a_{n}}+u(t)-\log (1-t) .
$$

Then $\hat{T}_{n}:=\min \left\{1 \leq i \leq n: X_{i}>\hat{a}_{n} v_{n}(i / n)+\hat{b}_{n}\right\}$ is an asymptotically optimal sequence of stopping times.

Remark 5.1. If $F$ is the distribution function of the standard normal distribution $N(0,1)$ then $F \in D(\Lambda)$ and normalization constants satisfying condition (5.6) are given by

$$
a_{n}=\frac{1}{\sqrt{2 \log n}}, \quad b_{n}=\sqrt{2 \log n}-\frac{\log \log n+\log 4 \pi}{2 \sqrt{2 \log n}} .
$$


Then possible choices of the constants $c_{n}$ and $d_{n}$ which satisfy (5.6) are

$$
c_{n}:=C(\log n)^{A}, \quad d_{n}:=D(\log n)^{B}
$$

with $A, B, C, D \in \mathbb{R}, A \geq 0, C>0$, and $B \leq A+\frac{1}{2}$. The limit constants $c$ and $d$ from (5.6) are given in this case by $c=-2 A$, and $d=-\sqrt{2} B D / C$ if $B=A+\frac{1}{2}$ and $d=0$ if $B<A+\frac{1}{2}$.

Proof of Theorem 5.1. By rearrangement,

$$
\frac{X_{i}-\hat{b}_{n}}{\hat{a}_{n}}=\frac{c_{i}}{c_{n}} \frac{Z_{i}-b_{n}}{a_{n}}-\frac{b_{n}}{a_{n}}\left(1-\frac{c_{i}}{c_{n}}\right)+\frac{d_{i}}{c_{n} a_{n}},
$$

and, thus,

$$
\hat{N}_{n}:=\sum_{i=1}^{n} \delta_{\left(i / n,\left(X_{i}-\hat{b}_{n}\right) / \hat{a}_{n}\right)}=\sum_{i=1}^{n} \delta_{R_{n}\left(i / n,\left(Z_{i}-b_{n}\right) / a_{n}\right)}
$$

with the transformation

$$
\begin{aligned}
R_{n}(t, y) & :=\left(t, \frac{c_{\lfloor t n\rfloor}}{c_{n}} y-\frac{b_{n}}{a_{n}}\left(1-\frac{c_{\lfloor t n\rfloor}}{c_{n}}\right)+\frac{d_{\lfloor t n\rfloor}}{c_{n} a_{n}}\right) \\
& \rightarrow R(t, y) \\
& :=\left(t, t^{c} y+d t^{c+1 / \alpha}\right) .
\end{aligned}
$$

For (5.7), note that $a_{\lfloor t n\rfloor} / a_{n} \rightarrow t^{1 / \alpha}$ (see Resnick (1987, Equation (0.18))) and, thus,

$$
\frac{d_{\lfloor t n\rfloor}}{c_{n} a_{n}}=\frac{d_{\lfloor t n\rfloor}}{c_{\lfloor t n\rfloor} a_{\lfloor t n\rfloor}} \frac{c_{\lfloor t n\rfloor}}{c_{n}} \frac{a_{\lfloor t n\rfloor}}{a_{n}} \rightarrow d t^{c+1 / \alpha} .
$$

Monotonicity of the constants implies that $R_{n}$ converges to $R$ uniformly on compact subsets in $(0,1] \times \mathbb{R}$ and $R$ maps $[0,1] \times(0, \infty]$ to $M_{\hat{f}}$ with $\hat{f}(t):=d t^{c+1 / d}$. The continuous mapping principle implies convergence of the point processes $\hat{N}_{n}$ to a Poisson process $\hat{N}$ on $M_{\hat{f}}$, where $\hat{N}$ has the intensity function

$$
\hat{G}(t, z)=G\left(R^{-1}(t, z)\right)=t^{c \alpha}\left(z-d t^{c+1 / \alpha}\right)^{-\alpha} \quad \text { on } M_{\hat{f}},
$$

where here $G(t, y)=y^{-\alpha}$ for $(t, y) \in[0,1] \times(0, \infty]$.

The intensity function $\hat{G}$ can be represented for $c+=1 / \alpha \neq 0$ in the form

$$
\hat{G}(t, z)=H\left(\frac{z}{v(t)}\right) \frac{v^{\prime}(t)}{v(t)}
$$

with $v(t):=t^{c+1 / \alpha}$ and $H(x):=(\alpha /(\alpha c+1))(x-d)^{-\alpha}$ for $x>d$. Theorem 5.1 implies convergence of the optimal stopping curves and stopping times of $\hat{N}_{n}$ to those of $\hat{N}$. The optimal stopping curve $u$ of $\hat{N}$ for the guarantee value has, by the results in Section 3 , for $c \neq-1 / \alpha$, the form given in (5.4). Thus, we obtain

$$
u(0)=\lim _{t \downarrow 0} \phi\left(\frac{1}{v(t)}\right) v(t)=\lim _{y \uparrow r} \frac{y}{\Phi(y)}=\lim _{y \uparrow r} y \exp \left(-\int_{d}^{y} \frac{1}{R(z)} \mathrm{d} z\right) .
$$

This implies Theorem 5.1, considering all cases one by one. 
Proof of Theorem 5.2. As in the previous proof, we calculate the intensity function of the limiting process as

$$
\hat{G}(t, z)=t^{-c \alpha}\left(-z+d t^{c-1 / \alpha}\right)^{\alpha}
$$

for $z<d t^{c-1 / \alpha}$ and 0 otherwise (which equals (3.11)).

To check that the uniform integrability condition (L) holds, we use the functions $v_{n}$ and their associated threshold stopping times

$$
\hat{T}_{n}(t):=\min \left\{t n<i \leq n: \frac{X_{i}}{\hat{a}_{n}}>v_{n}\left(\frac{i}{n}\right)\right\}, \quad \hat{T}_{n}:=\hat{T}_{n}(0) .
$$

Convergence of $\lambda_{n}(t):=c_{\lfloor t n\rfloor} / c_{n} \rightarrow t^{c}$ and $\mu_{n}(t):=d_{\lfloor t n\rfloor} / c_{n} a_{n} \rightarrow d t^{c+1 / \alpha}$ follow by the monotonicity conditions and by continuity of the limiting functions uniform on each interval $[t, 1], t>0$. Furthermore, $\lim _{t \uparrow 1} u_{c, 0}(t) / u_{0,0}(t)=1$ and, by $(3.14), \lim _{t \uparrow 1} u_{c, d}(t)-u_{c, 0}(t)=$ $d$. This is the basic tool for establishing the uniform integrabilty condition (L). For details of the proof, see Faller (2009).

Proof of Theorem 5.3. The proof is analogous to the previous proofs. With the constants $\hat{b}_{n}:=c_{n} b_{n}+d_{n}$ we obtain in the limit the transformation $R(t, y)=(t, y-c \log t-d \log t)$. Thus, $R^{-1}(t, z)=(t, z+(c+d) \log t)$ and the intensity function of the limit process $\hat{N}$ is given by

$$
\hat{G}(t, z)=G\left(R^{-1}(t, z)\right)=\mathrm{e}^{-z} t^{-(c+d)},
$$

where here $G(t, y)=\mathrm{e}^{-y}$. The optimal stopping curve of $\hat{N}$ is given by

$$
u(t)=\log \left(\frac{1}{1-(c+d)}\left(1-t^{1-(c+d)}\right)\right) .
$$

For details of the proof of the uniform integrability condition $(\mathrm{L})$, we refer the reader to Faller (2009).

\section{References}

Bruss, F. T. AND Rogers, L. C. G. (1991). Embedding optimal selection problems in a Poisson process. Stoch. Process. Appl. 38, 267-278.

Chow, Y. S., Robins, H. AND Siegmund, D. (1971). Great Expectations: The Theory of Optimal Stopping. Houghton Mifflin, Boston, MA.

De Haan, L. and Verkadde, E. (1987). On extreme-value theory in the presence of a trend. J. Appl. Prob. 24, 62-76.

FAller, A. (2009). Approximative Lösungen von Mehrfachstoppproblemen. Doctoral Thesis, University of Freiburg.

FAller, A. ANd RÜSCHENDORf, L. (2011). On approximative solutions of multistopping problems. Ann. Appl. Prob. 21, 1965-1993.

GNedin, A. V. AND SAKAgUCHI, M. (1992). On a best choice problem related to the Poisson process. In Strategies for Sequential Search and Selection in Real Time (Amherst, MA, 1990; Contemp. Math. 125), American Mathematical Society, Providence, RI, pp. 59-64.

Kennedy, D. P. AND Kertz, R. P. (1990). Limit theorems for threshold-stopped random variables with applications to optimal stopping. Adv. Appl. Prob. 22, 396-411.

Kennedy, D. P. AND KeRTz, R. P. (1991). The asymptotic behavior of the reward sequence in the optimal stopping of i.i.d. random variables. Ann. Prob. 19, 329-341.

KüHne, R. AND Rüschendorf, L. (2000a). Approximation of optimal stopping problems. Stoch. Process. Appl. 90, $301-325$

KüHne, R. AND Rüschendorf, L. (2000b). Optimal stopping with discount and observation costs. J. Appl. Prob. 37, 64-72.

KÜHNE, R. AND RÜSCHENDORF, L. (2003). Optimal stopping and cluster point processes. Statist. Decisions 21, $261-282$.

KüHnE, R. And Rüschendorf, L. (2004). Approximate optimal stopping of dependent sequences. Theory Prob. Appl. 48, 465-480.

Resnick, S. I. (1987). Extreme Values, Regular Variation, and Point Processes. Springer, New York. 